\title{
Cirurgia plástica após grande perda ponderal
}

\section{Plastic surgery after massive weight loss}

Fernando SAnfelice André ${ }^{1}$

Trabalho realizado no Hospital Regional Hans Dieter Schmidt, Joinville, SC, Brasil.

Artigo submetido pelo SGP (Sistema de Gestão de Publicações) da RBCP.

Artigo recebido: 8/2/2010 Artigo aceito: $16 / 5 / 2010$

\section{RESUMO}

O autor apresenta nesse artigo, suas condutas, táticas e técnicas cirúrgicas utilizadas no tratamento dos pacientes ex-obesos mórbidos que obtiveram grande perda ponderal, submetidos ou não à gastroplastia. Relatamos complicações, intercorrências, resultados obtidos e as conclusões de 220 procedimentos adotados.

Descritores: Cirurgia plástica. Obesidade mórbida. Gastroplastia.

\section{SUMMARY}

The author presents in this paper, his conduct, tactical and surgical techniques, used in the treatment of patients after massive weight loss, submitted or not to gastroplasty. Complications, results and conclusions of 220 procedures are reported.

Descriptors: Plastic surgery. Morbid obesity. Gastroplasty.

\section{INTRODUÇÃO}

A cirurgia plástica dos ex-obesos mórbidos apresenta-se como um novo e amplo campo de atuação para o cirurgião plástico moderno.

Inúmeras deformidades resultantes da brutal redução ponderal se fazem presentes. As principais deformidades são as seguintes: 1) grande excesso cutâneo vertical e horizontal do abdome, com hérnias incisionais frequentes (Figura 1);2) flacidez e atrofia mamária (Figura 2); 3 ) flacidez das coxas (Figura 3); 4) flacidez dos braços (Figura 4); 5) flacidez cutânea do dorso (Figura 5); 6) ptose das nádegas; 7) ptose do púbis (Figura 6).

Diversos autores descreveram suas condutas para o tratamento destes pacientes ${ }^{1-21}$. Nesse artigo, o autor apresenta suas condutas, resultados e conclusões dos procedimentos adotados para esses pacientes. Todas as cirurgias foram realizadas unicamente pelo autor.

\section{MÉTODO}

No período de janeiro de 2006 a fevereiro de 2010 , foram realizadas 220 cirurgias em pacientes ex-obesos, submetidos ou não à gastroplastia. Todos os pacientes foram submetidos a exames laboratoriais (hemograma, glicemia, coagulograma, proteínas totais e frações, ureia, creatinina, cálcio, ferro sérico e ferritina), avaliação cardiológica (eletrocardiograma e radiografia de tórax) e anestesiológica. Os pacientes submetidos à gastroplastia só tiveram sua cirurgia programada após comprovação de 1 ano da cirurgia anterior, peso estabilizado há 3 meses, índice de massa corpórea (IMC) abaixo de 30 e liberação da equipe multidisciplinar (psicólogo, nutricionista, cirurgião bariátrico, cirurgião plástico). Os pacientes que apresentavam abdome em avental ou flacidez crural importante, limitando a atividade diária e dificultando a progressão da redução ponderal, foram exceção. Nesses pacientes foram realizadas: abdominoplastia em âncora ou cruroplastia, consideradas cirurgias higiênicas (todos os pacientes com IMC acima de 30). Os pacientes que apresentaram grande perda ponderal, sem cirurgia bariátrica, puderam ser submetidos à cirurgia sem as exigências do outro grupo (pós-gastroplastia), exceto quanto à normalidade dos exames pré-operatórios e à avaliação anestesiológica.

As cirurgias realizadas foram:

- abdominoplastia clássica ou em âncora; tratamento de hérnia incisional (quando presente), eventual uso de tela sintética;

- mamaplastia (vertical, L, LT, T, T com enxerto do CAM, $\mathrm{T}$ com retalho de pedículo inferior areolado), com o uso de implantes em casos específicos;

- cruroplastia, com cicatriz na linha da virilha, ou estendida até o joelho, de acordo com o caso; braquioplastia

1. Membro Titular da Sociedade Brasileira de Cirurgia Plástica; Cirurgião Plástico do Hospital Regional Hans Dieter Schmidt, Joinville, SC. 
(convencional ou estendida, da axila até o sulco mamário inferior);

- lifting de nádegas;

- lifting de púbis (simultaneamente à abdominoplastia). Em casos selecionados, foi necessário um segundo tempo cirúrgico para alcançarmos um resultado ideal do formato pubiano (pacientes com extrema flacidez e/ou excesso adiposo).

Os seguintes procedimentos foram empregados rotineiramente:

- drenagem a vácuo com dreno tipo suctor, até que o volume drenado fosse menor que $50 \mathrm{ml} / \mathrm{dia}$, nas abdominoplastias e cruroplastias;

- antibioticoterapia (cefazolina endovenosa, $2 \mathrm{~g}$ intraoperatoriamente e $4 \mathrm{~g} / \mathrm{dia}$, durante a permanência hospitalar; Cefalexina $2 \mathrm{~g} /$ dia por mais 5 dias após a alta hospitalar);

- curativos diários durante a internação e a cada 3 dias, por 60 dias após a alta hospitalar (fita microporosa);

- drenagem com dreno Penrose nas mamaplastias e nas braquioplastias, por 1 ou 2 dias, de acordo com o volume drenado;

- mobilização precoce, deambulação no $1^{\circ}$ dia de pósoperatório, em todos os casos;

- sondagem vesical de demora por 24 horas, nas abdominoplastias e cruroplastias;

- compressão elástica abdominal, braquial e de coxas no $1^{\circ}$ dia de pós-operatório; sutiãs no $1^{\circ}$ dia de pós-operatório, nas mamaplastias;

- não utilizamos anticoagulantes de rotina; exceto nos casos em que havia indicação obrigatória;

- utilizamos os seguintes fios de sutura: mononylon 3-0 para a montagem dos retalhos e suturas profundas;
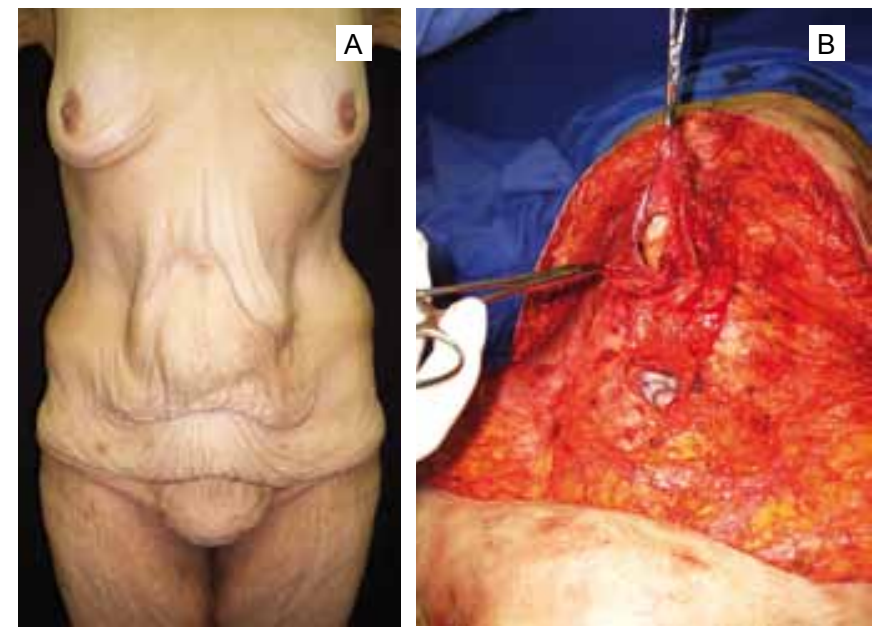

Figura 1 - A: flacidez do abdome; B: hérnia incisional. mononylon 4-0 para as suturas do subcutâneo, na montagem do CAM, na axila, na cicatriz umbilical e na linha da virilha; e o monocryl 4-0 para suturas finais da pele em nível intradérmico. Os pontos externos de mononylon foram retirados após 10 dias de pós-operatório (cicatriz umbilical, axila, região inguinal, CAM).

\section{RESULTADOS}

Apesar das complicações e intercorrências (Figura 7), nossos pacientes apresentaram bons resultados estéticos e funcionais (Figuras 8 a 14).

Apesar de todo cuidado tomado na realização das cirurgias, adotando-se todos os preceitos da Cirurgia Plástica, para obtermos os melhores resultados possíveis, tivemos complicações e intercorrências, listadas abaixo:

- seromas (abdominais ou de coxas) em 10 casos;

- hematoma (abdominal ou de mama) em 2 casos;

- deiscências parciais de sutura abdominais em 4 casos; de coxas em 1 caso;
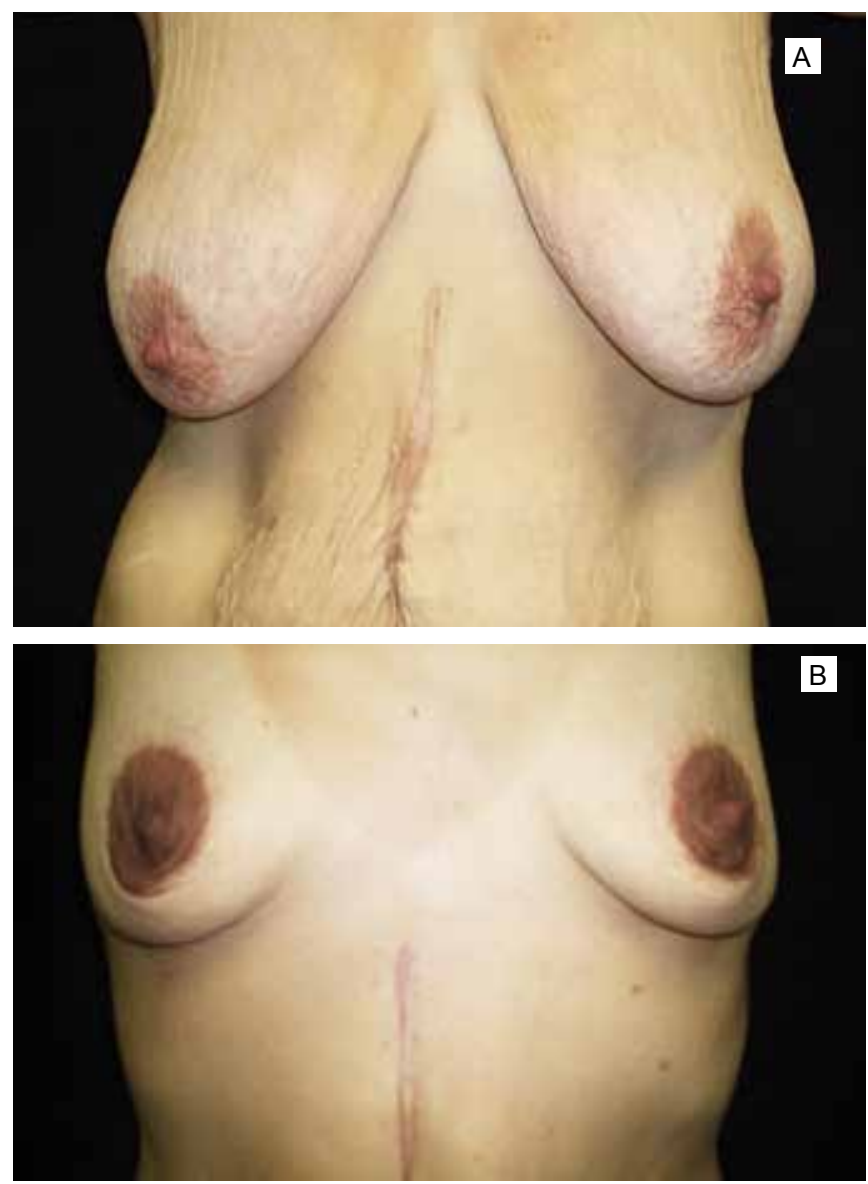

Figura 2 - A: flacidez mamária; B: atrofia mamária. 
- não tivemos nenhum caso de infecção;

- trombose venosa profunda em 1 caso, unilateral, 60 dias após lifting crural;

- necroses parciais da extremidade dos retalhos abdominais em 4 casos e de coxas em 1 caso;

- assimetria mamária em 1 caso, que foi reoperado;

- correção de cicatrizes hipertróficas em 5 casos (braquiais 3, abdominais 2);

- correção de alargamento da cicatriz umbilical: 10 casos.

\section{DISCUSSÃO}

O tratamento do ex-obeso é desafiador para o cirurgião plástico, na atualidade. Alguns aspectos podem causam temor na realização dessas cirurgias, principalmente naqueles pacientes submetidos à gastroplastia (anemias crônicas, níveis anormais de proteínas, vitaminas e oligoelementos,

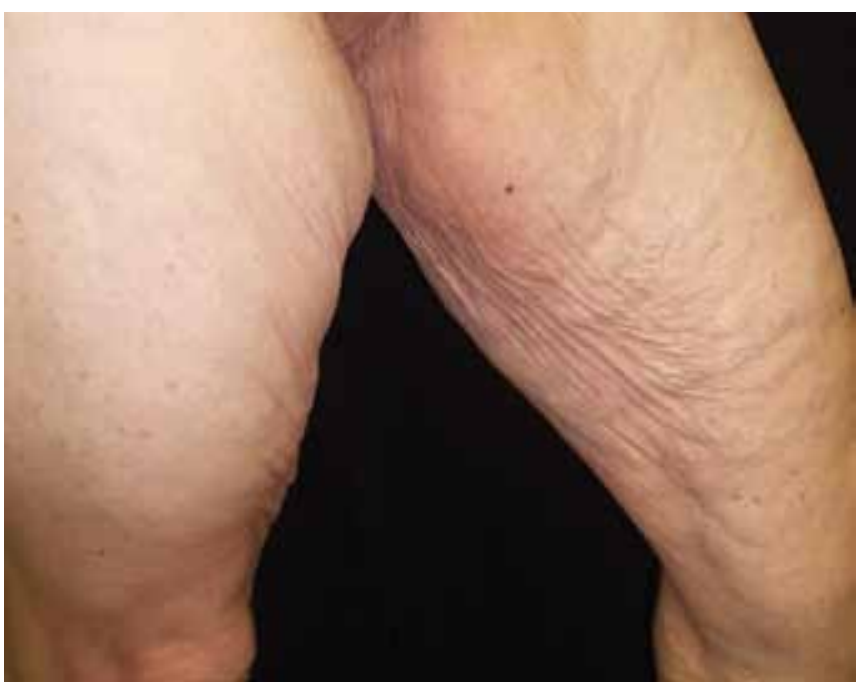

Figura 3 - Flacidez de coxa.

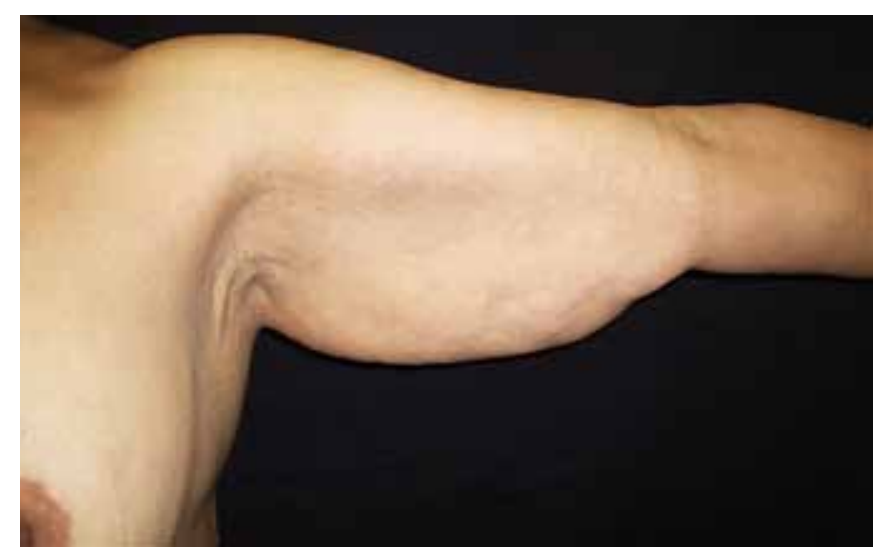

Figura 4 - Flacidez do braço.

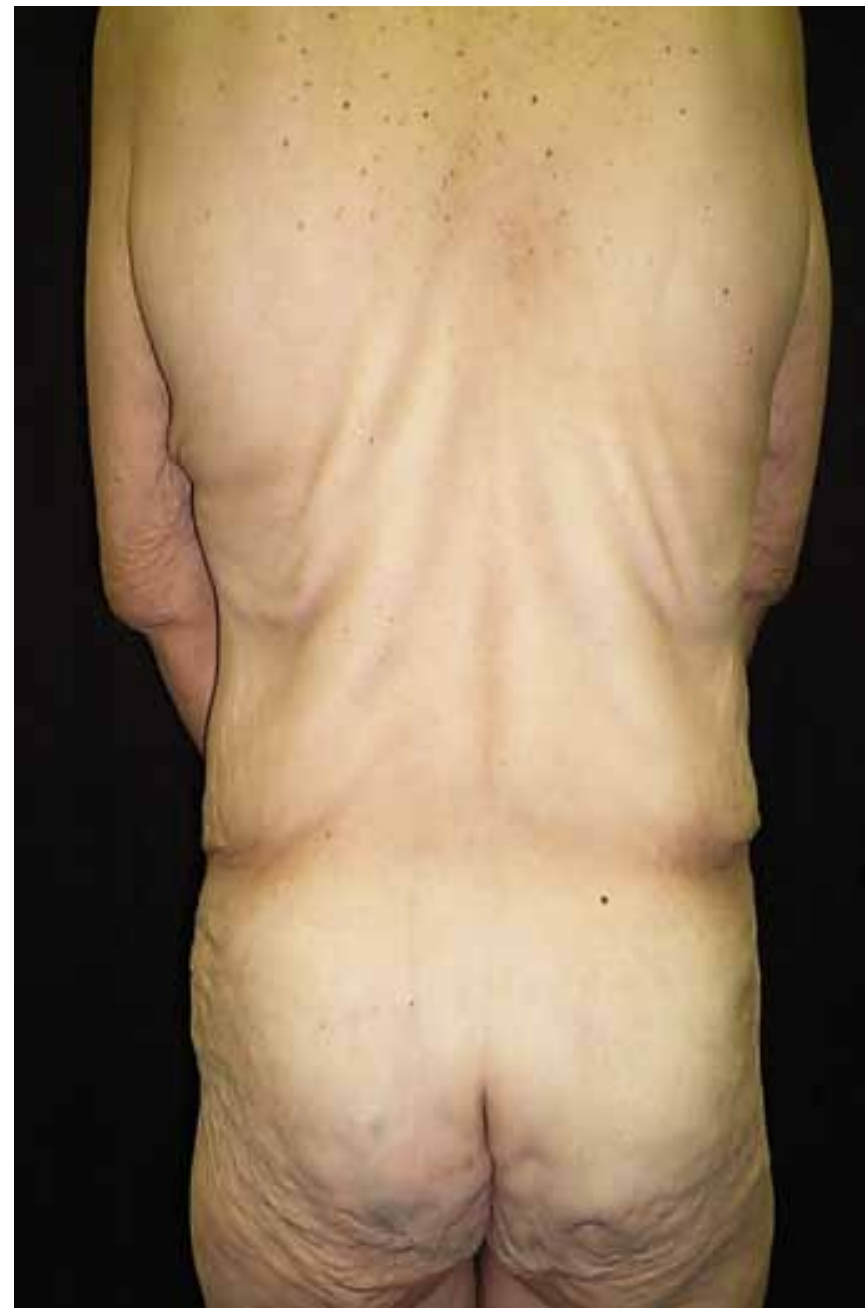

Figura 5 - Flacidez do dorso e nádegas.

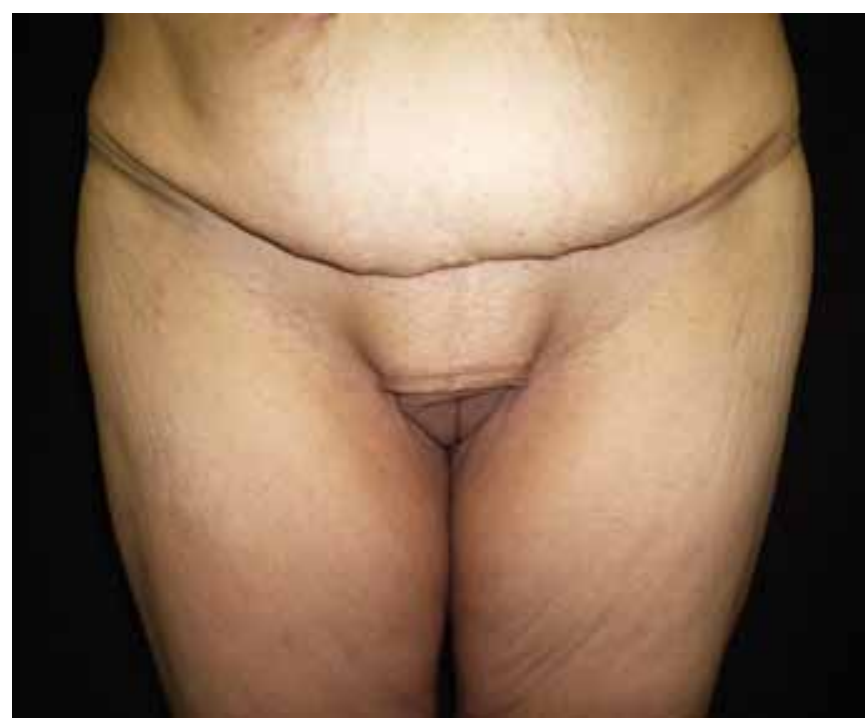

Figura 6 - Ptose do púbis. 

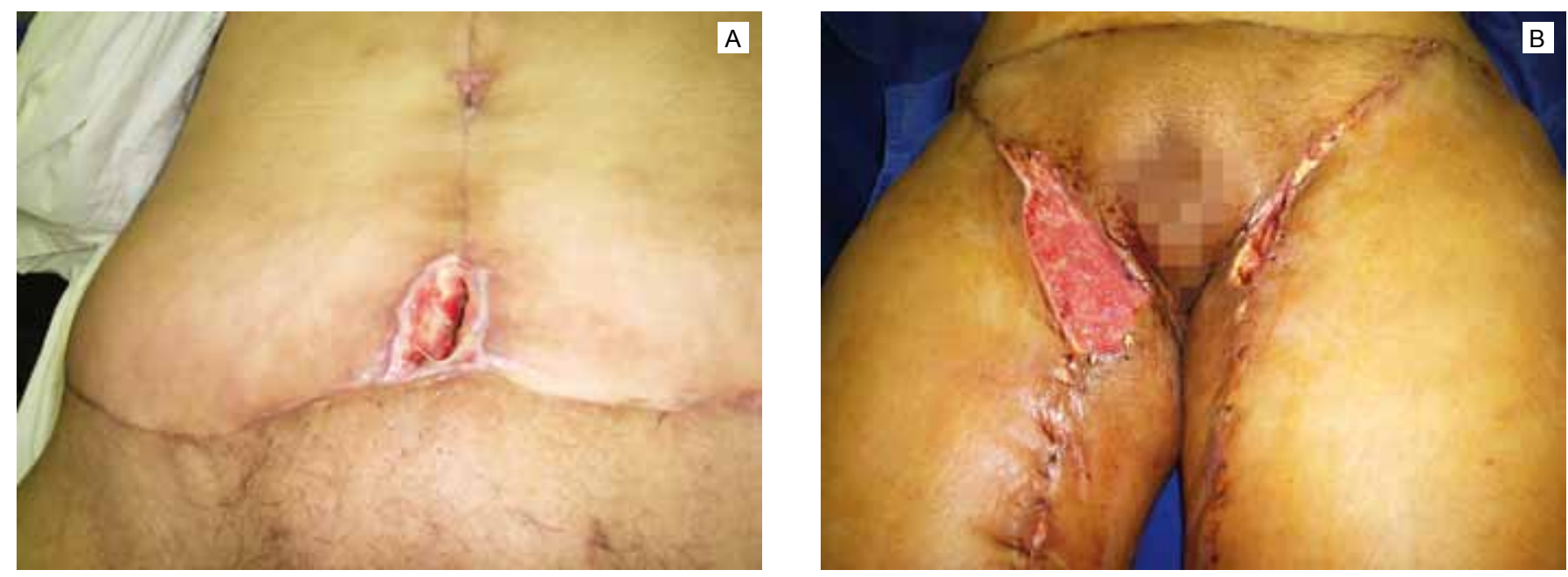

Figura 7 - A: necrose da extremidade do retalho abdominal; B: extensa deiscência de sutura em cruroplastia.

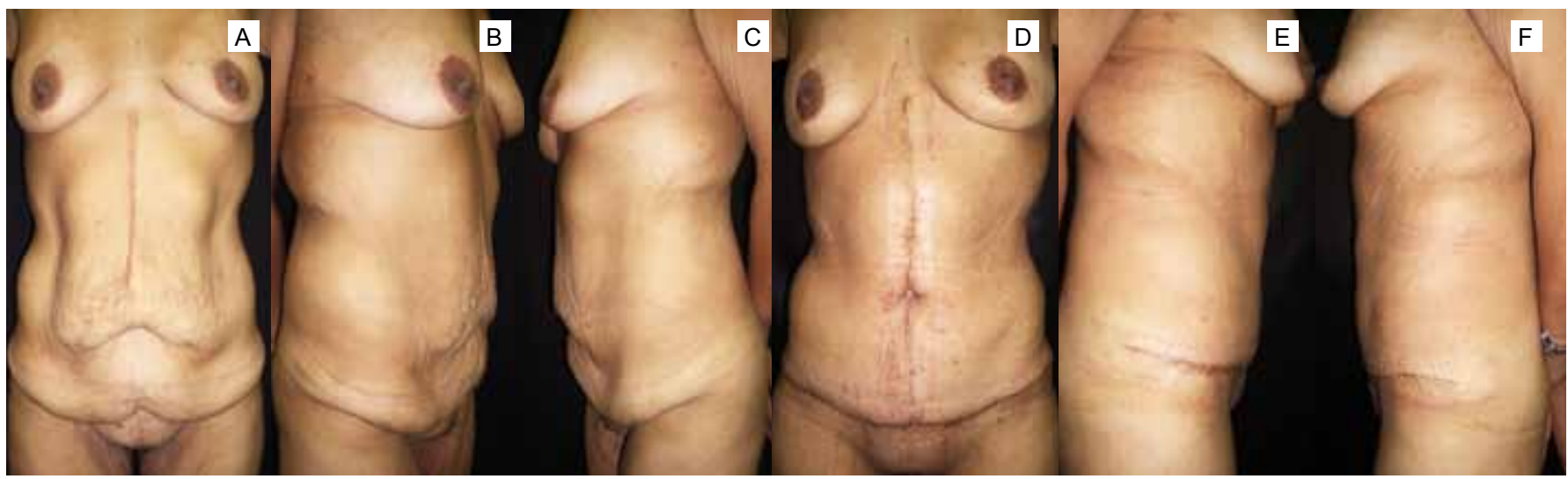

Figura 8 - A: abdome, aspecto pré-operatório, vista ântero-posterior; B: abdome, aspecto pré-operatório, vista oblíqua direita; $\boldsymbol{C}:$ abdome, aspecto pré-operatório, vista oblíqua esquerda; D: abdome, aspecto pós-operatório, vista ântero-posterior; $\mathbf{E}:$ abdome, aspecto pósoperatório, vista oblíqua direita; $\boldsymbol{F}$ : abdome, aspecto pós-operatório, vista oblíqua esquerda.

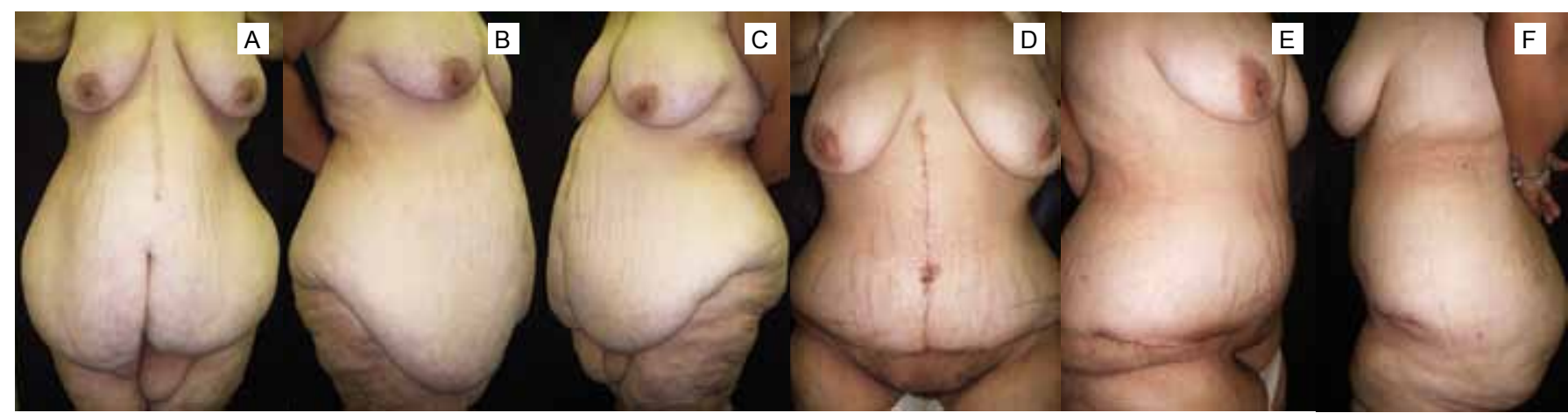

Figura 9 - A: abdome, aspecto pré-operatório, vista ântero-posterior; B: abdome, aspecto pré-operatório, vista oblíqua direita; $\boldsymbol{C}:$ abdome, aspecto pré-operatório, vista obliqua esquerda; D: abdome, aspecto pós-operatório, vista ântero-posterior; $\boldsymbol{E}:$ abdome, aspecto pósoperatório, vista oblíqua direita; $\boldsymbol{F}$ : abdome, aspecto pós-operatório, vista oblíqua esquerda. 


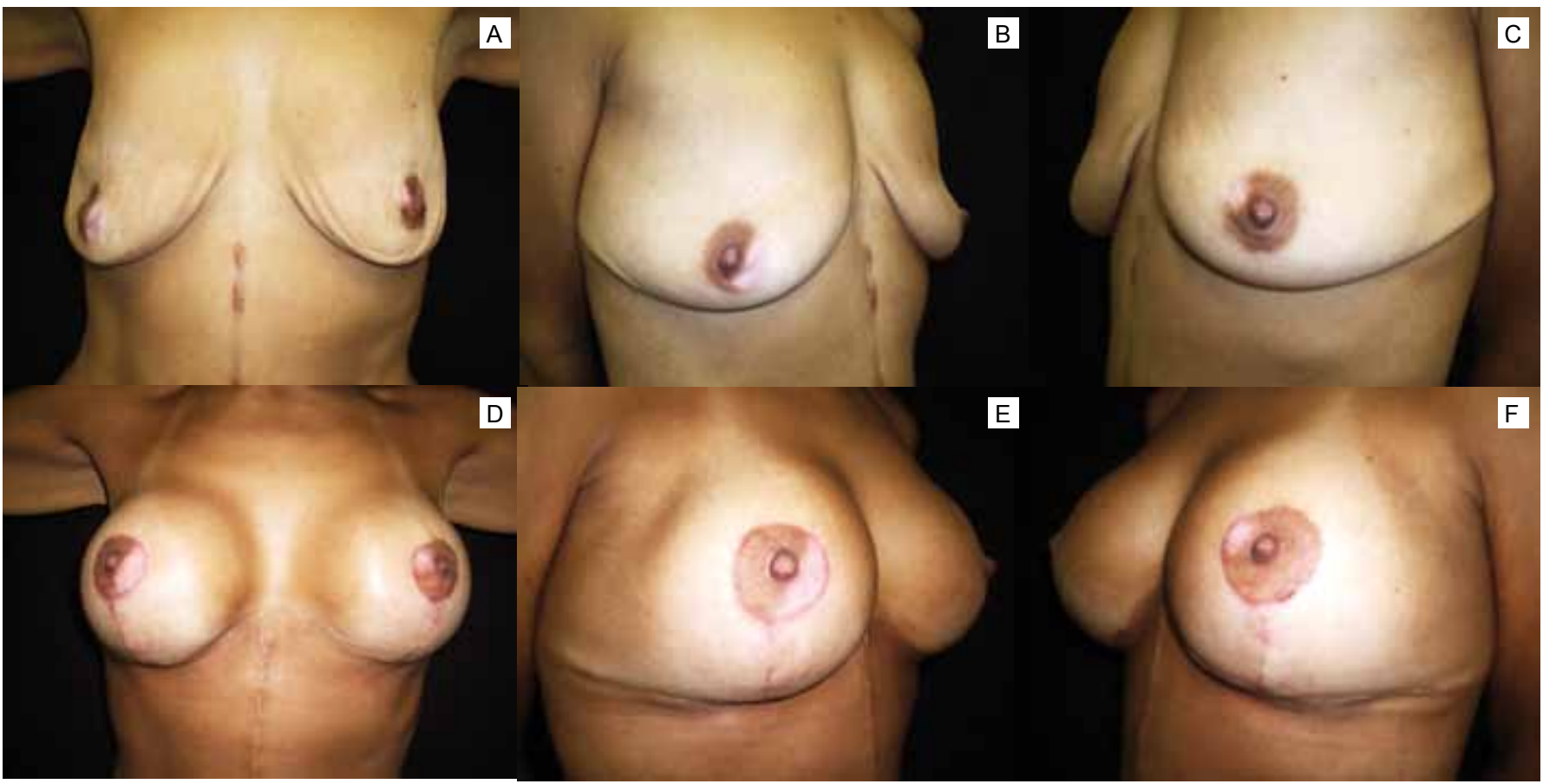

Figura 10 - A: mamas, aspecto pré-operatório, vista ântero-posterior; B: mamas, aspecto pré-operatório, vista obliqua direita; $\boldsymbol{C}$ : mamas, aspecto pré-operatório, vista oblíqua esquerda; D: mamas, aspecto pós-operatório, vista ântero-posterior; E: mamas, aspecto pósoperatório, vista oblíqua direita; $\boldsymbol{F}$ : mamas, aspecto pós-operatório, vista oblíqua esquerda.

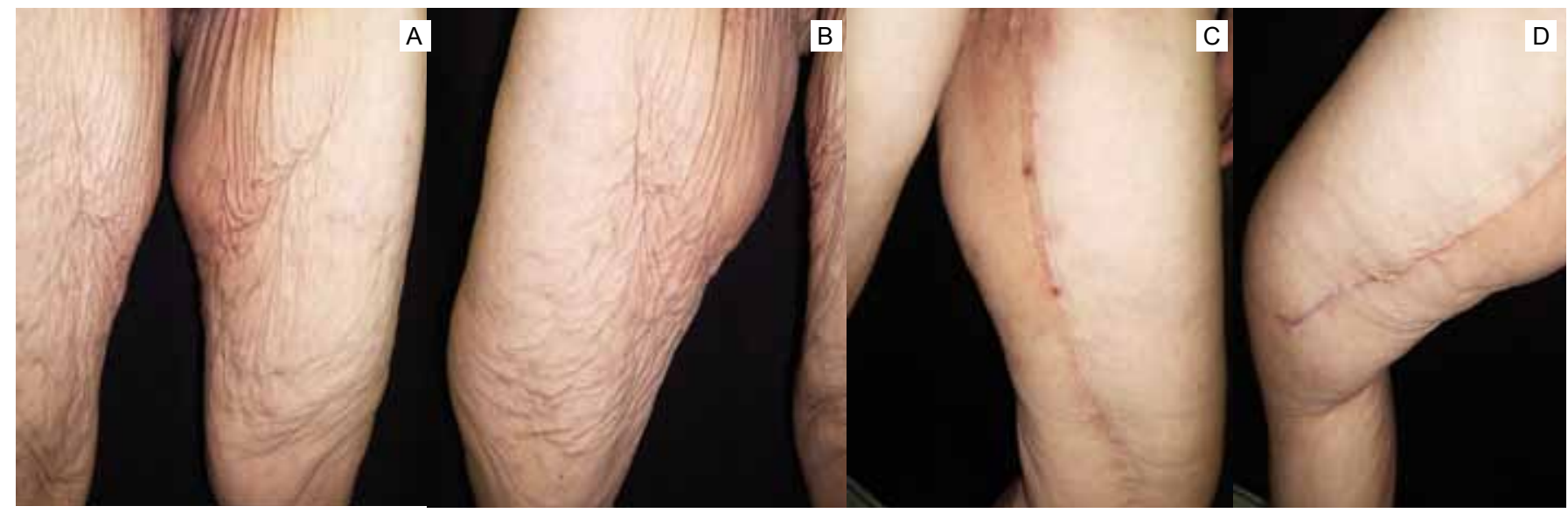

Figura 11 - A: coxa esquerda, aspecto pré-operatório; B: coxa direita, aspecto pré-operatório; $\boldsymbol{C}$ : coxa esquerda, aspecto pós-operatório; D: coxa direita, aspecto pós-operatório.

maior sangramento, entre outros). Devemos ser mais criteriosos ainda no momento da indicação cirúrgica. Cirurgias múltiplas ou combinadas não foram indicadas, devido ao prolongado tempo cirúrgico e consequente aumento, inaceitável para nós, do risco de complicações no pós-operatório.

Notamos que os pacientes submetidos à gastroplastia, comparados aos pacientes não submetidos à cirurgia, têm um índice muito maior de seromas (abdome, coxas), sendo quase uma regra. Os seromas nesses pacientes exigiram maior número de punções e o volume drenado muitas vezes maior. Em um caso de abdominoplastia, chegamos a drenar $800 \mathrm{ml}$ em uma única sessão. Mesmo utilizando os pontos de adesão do retalho abdominal à aponeurose dos músculos retos, conforme prescrito por Baroudi ${ }^{6}$, ainda assim tivemos casos de seromas.

Nos pacientes que apresentaram seromas recorrentes e de 


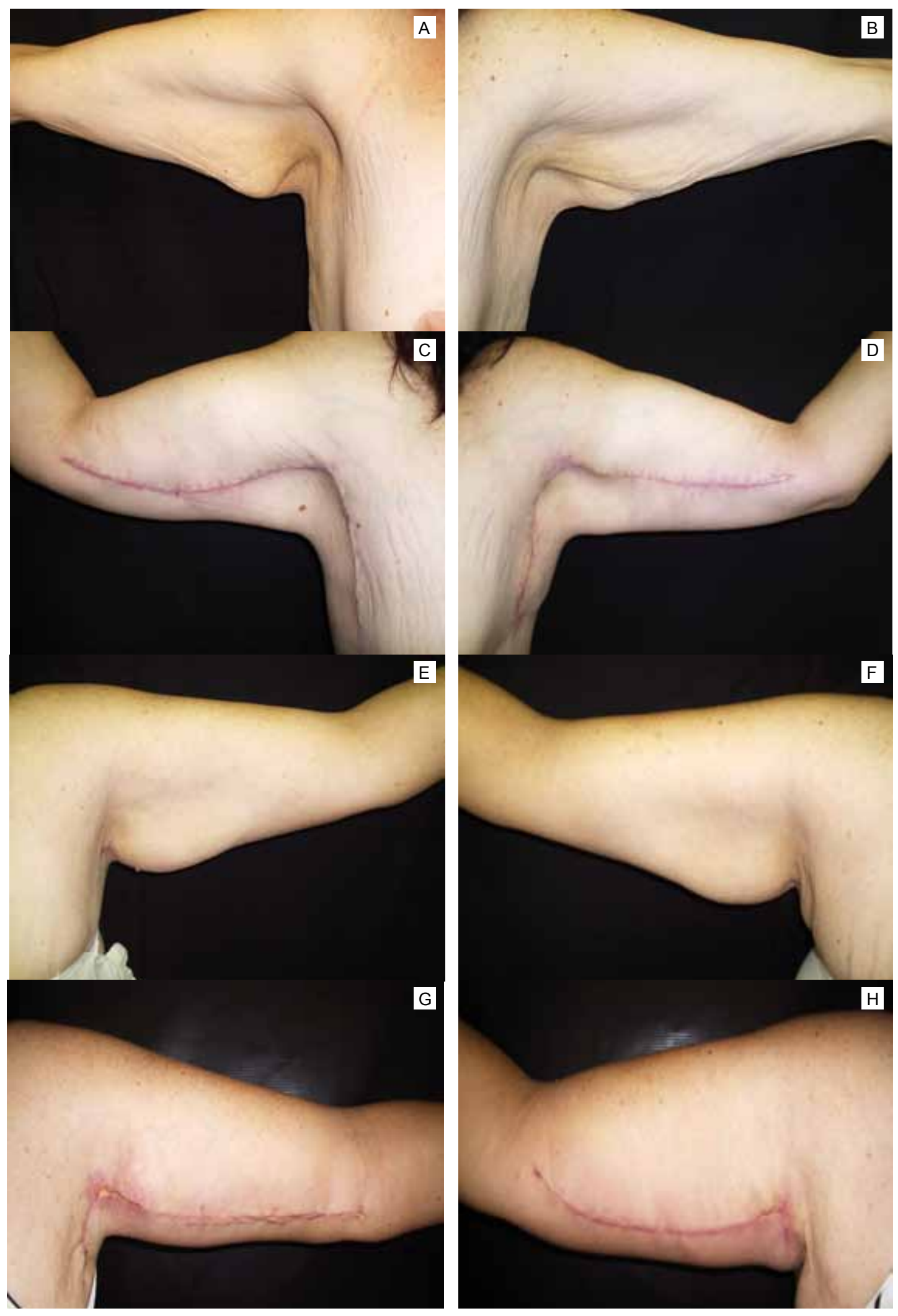

Figura 12 - A: braço direito, aspecto pré-operatório; B: braço esquerdo, aspecto pré-operatório; C: braço direito, aspecto pós-operatório; D: braço esquerdo, aspecto pós-operatório; $\boldsymbol{E}$ : pré-operatório; $\boldsymbol{F}$ : pré-operatório; G: pós-operatório; H: pós-operatório. 


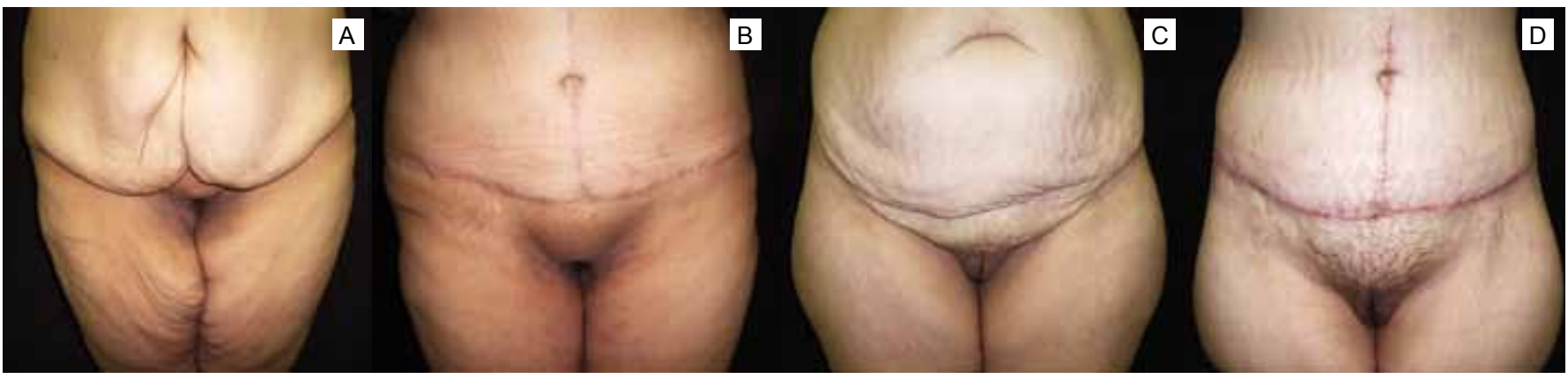

Figura 13 - A: púbis, aspecto pré-operatório; B: púbis, aspecto pós-operatório; $\boldsymbol{C}$ : púbis, aspecto pré-operatório; D: púbis, aspecto pós-operatório.
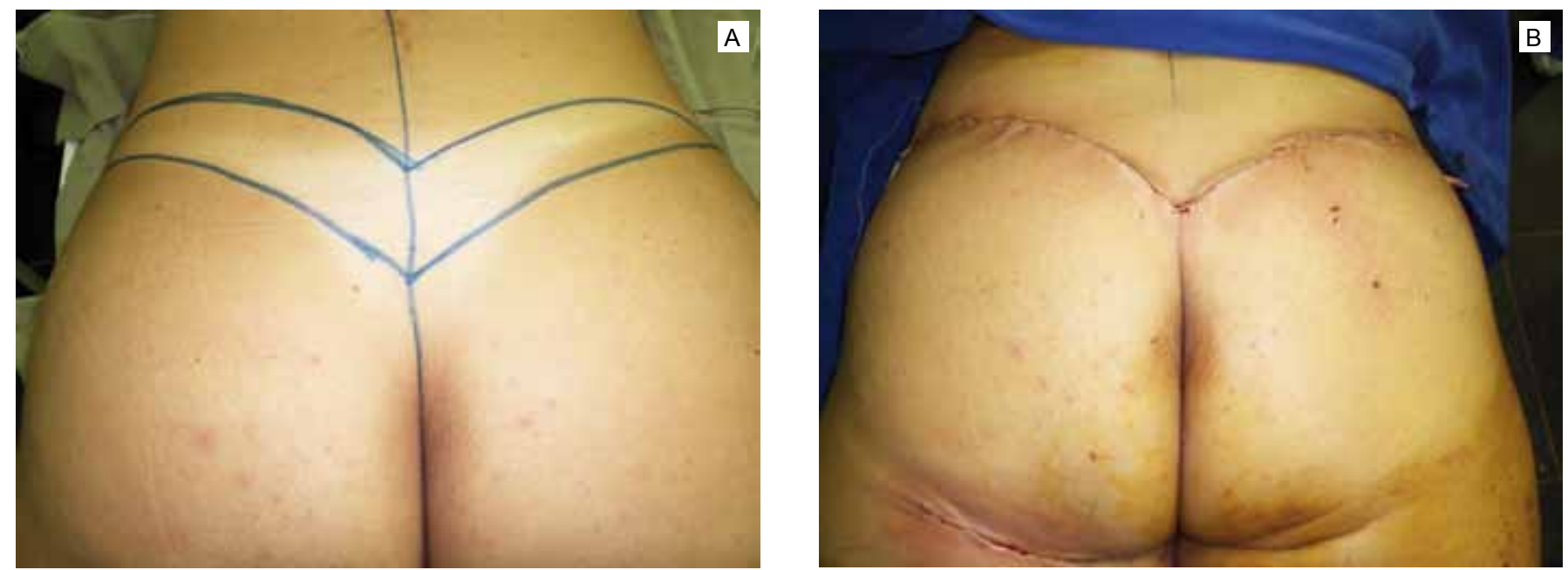

Figura 14 - A: glúteos, aspecto pré-operatório; B: glúteos, aspecto pós-operatório.

grande volume drenado, a espessura do panículo adiposo era muito relevante, pois se tratavam de pacientes submetidos a cirurgias higiênicas. Os pacientes ex-obesos não submetidos à gastroplastia, não importando o grau de redução ponderal, foram menos propensos a complicações ou intercorrências, pois suas condições clínicas eram superiores ao outro grupo. Os casos de deiscências de sutura foram intimamente relacionados com as necroses da extremidade dos retalhos, ou seja, se não ocorreu necrose, não houve deiscência de sutura.

A grande diversidade das dismorfias corporais encontradas nos ex-obesos exigiu muito tempo de estudo e planejamento. As deformidades abdominais exigiram duas condutas, cirurgia em âncora ou clássica, eventualmente associadas à herniorrafia incisional, convencional ou com uso de tela sintética. As mamas exigiram sete condutas diferentes, de acordo com a forma mamária encontrada. As deformidades das coxas e braços exigiram duas abordagens distintas (cicatrizes ao nível inguinal ou estendendo-se até o joelho; justa axilar ou até o cotovelo, de acordo com o caso).

As deformidades do púbis foram tratadas conjuntamente com o abdome. Eventualmente, um segunto tempo cirúrgico foi necessário, devido ao importante grau de flacidez e ptose do mesmo. As nádegas foram tratadas em procedimento isolado.

\section{CONCLUSÃO}

A conduta adotada até o momento tem se mostrado correta e segura. Não tivemos nenhuma complicação ou intercorrência que prejudicasse o resultado final do tratamento, mesmo com a exigência de retoques nas cicatrizes ou nos casos de um segundo tempo cirúrgico. Pudemos proporcionar resultados estéticos e funcionais muito satisfatórios, além de melhorar a qualidade de vida dos pacientes. Ressaltamos que o cirurgião plástico que abraçar esse novo campo de atuação, deve ter amplo domínio de todas as técnicas e táticas cirúrgicas a serem utilizadas, assim como saber lidar com as complicações e intercorrências. O cirurgião plástico deve ter, também, total apoio de uma equipe multidisciplinar. O tratamento dos ex-obesos é mais complexo do que pode parecer a princípio, requer muito 
mais cuidado no planejamento cirúrgico, na condução do pré e pós-operatório, quando comparados aos pacientes não ex-obesos. Os pacientes ex-obesos constituem um novo grupo, totalmente diferente daquele que se apresenta na nossa clínica diária.

\section{REFERÊNCIAS}

1. Baroudi R. Flankplasty: a specific treatment to improve body contouring. Ann Plast Surg. 1991;27(5):404-20.

2. Lockwood T. Brachioplasty with superficial facial system suspension. Plast Reconstr Surg. 1995;94(4):912-20.

3. Hustad JP. Body contouring in obese patient. Clin Plast Surg. 1996;23(4):647-70.

4. Soundararajan V, Hart NB, Royston CM. Abdominoplasty following vertical banded gastroplasty for morbid obesity. Br J Plast Surg. 1995;48(6):423-7.

5. Le Louarn C, Pascal JF. High superior tension abdominoplasty. Aesthetic Plast Surg. 2000;24(5):375-81.

6. Baroudi R, Ferreira CA. Seroma: how to avoid it and how to treat it. Aesthet Surg J. 1998;18(6):439-41.

7. Bozola AR, Psillakis JM. Abdominoplasty: a new concept and classification for treatment. Plast Reconstr Surg. 1988;82(6):983-93.

8. Soundararajan V, Hart NB, Royston CM. Abdominoplasty following vertical banded gastroplasty for morbid obesity. J Plast Surg. 1995;48(6):423-7.

9. Kaluf R, Araújo GAZ, Martins DL, Campos LCB, Magalhães RC, Santos Júnior AJ. Tratamento da região pubiana na abdominoplastia de pacientes após grande perda ponderal. Rev Bras Cir Plást.
2008;23(4):302-4.

10. Roxo CDP, Pinheiro O, Almeida D. Abdominoplastia multifuncional. Rev Soc Bras Cir Plast. 2004;19(3):54-74.

11. Borud LJ, Warren AG. Modified vertical abdominoplasty in the massive weight loss patient. Plast Reconstr Surg. 2007;119(6):1911-21.

12. Hurwitz DJ, Zewert T. Body contourning after bariatric surgery. Op Tech Plast Surg. 2002;(1):87-97.

13. Cintra W Jr, Modolin ML, Gemperli R, Gobbi CI, Faintuch J, Ferreira MC. Quality of life after abdominoplasty in women after bariatric surgery. Obes Surg. 2008;18(6):728-32.

14. Wallach SG. Abdominal contour surgery for the massive weight loss patient: the fleur-de-lis approach. Aesthet Surg J. 2005;25(5):454-65.

15. Savage RC. Abdominoplasty following gastrointestinal bypass surgery. Plast Reconstr Surg. 1983;71(4):500-9.

16. Roxo del Pino C, Roxo Weck AC, Pinto de Aguiar Becerra E, Labanca L, Martins Pinto C, Braquioplastia: abordaje simple. Cir Plást Iberolatinam. 2009;35(3):171-80.

17. André FS. Abdominoplastia e lifting do púbis. Rev Bras Cir Plást. 2009;24(3):345-50

18. André FS. Mamaplastia pós-grande perda ponderal. Rev Bras Cir Plást 2009;24(4):441-9.

19. Bozzola AR, Bozzola Filho Í, Freitas R. Suspensão composta glúteocrural. Rev Bras Cir Plást. 2008;23(3):189-91.

20. Roxo CDP, RoxoACW, Labanca L, Martins CRP. Tratamento das hérnias incisionais nas abdominoplastias multifuncionais. Rev Bras Cir Plást. 2008;23(3):184-8.

21. Souza MCA, Silva CSC. Body lift: tratamento do contorno corporal em pacientes ex-obesos. Rev Bras Cir Plást. 2008;23(4):294-301.

Correspondência para:

Dr. Fernando Sanfelice André

Rua Osvaldo Cruz, 128 - Boa Vista - Joinville, SC, Brasil - CEP: 89205-240

E-mail: sanfi@brturbo.com.br 